\title{
Characterisation of the contact pressure dependent interfacial heat transfer coefficient for a hot stamping process following a data driven approach
}

\author{
Xiaochuan Liu ${ }^{1}$, Yang Zheng ${ }^{1}$, Omer El Fakir ${ }^{1}$, Rajab Said ${ }^{2}$ and LiLiang Wang, ${ }^{1, *}$ \\ ${ }^{1}$ Department of Mechanical Engineering, Imperial College London, London, SW7 2AZ, UK \\ ${ }^{2}$ ESI Group, Paris, 75015, France
}

\begin{abstract}
In the present research, the contact pressure characteristics of a hot stamping process was revealed by data mining an existing data base of verified FE simulation results, collected by a cloud-based finite element (FE) simulation platform. Following a data driven approach, a dedicated, novel testing scheme for a hot stamping process was developed and applied to determine the effect of contact pressure on the interfacial heat transfer coefficient (IHTC) between an AA7075 aluminium alloy and cast-iron tools. In this novel testing scheme, only one single IHTC test was required which has improved testing efficiency significantly.
\end{abstract}

Keywords: Hot stamping, Aluminium, Interfacial heat transfer coefficient

\section{Introduction}

Aluminium alloys have been widely applied in the sheet hot stamping industry, and their heat transfer characteristics in such processes require a more thorough understanding. The interfacial heat transfer coefficient (IHTC) is one of the most important heat transfer characteristics that determines the cooling rate of a component in a hot stamping process. When the cooling rate is above the critical value, the full mechanical strength of the formed component can be retained after the artificial ageing process [1]. Therefore, the IHTC should be determined and then implemented in finite element (FE) simulations to precisely simulate the temperature evolution and cooling rate of the component during the hot stamping process.

Great efforts have been made to determine the IHTC at different contact pressures, which is one of the most important influential factors that affects the IHTC. In the study of Chang et al. [2], the IHTC between a boron steel and a tool steel was determined at different contact pressures of $0.006,0.04,1,5,15$ and $25 \mathrm{MPa}$, and found to increase exponentially with increasing contact pressure from approximately 0.3 to $4.6 \mathrm{~kW} / \mathrm{m}^{2} \mathrm{~K}$. A linearly increasing trend of the IHTC with contact pressure was obtained by applying different contact pressures of 4,8 , 15 and $24 \mathrm{MPa}$, in the study of Caron et al [3]. Another eight different contact pressures were selected from 0 to $24 \mathrm{MPa}$ and applied to investigate the effect of contact pressure on the IHTC between an aluminium alloy and a cast-iron steel by Liu et al. [4]. It was found that the IHTC increased logarithmically with the increasing contact pressure from approximately 0.8 to $15.1 \mathrm{~kW} / \mathrm{m}^{2} \mathrm{~K}$. Larger contact pressures of 30 and $40 \mathrm{MPa}$ were used to determine the IHTC values for an titanium alloy in hot stamping processes in the study of Zhang et al. [5].

In previous studies, different static contact pressure conditions were selected based on experience to determine the IHTC values, which would not satisfy the exact contact pressure conditions encountered in hot stamping processes, leading to a decrease in testing efficiency. In addition, the applied contact pressure may change over extremely short time periods in hot stamping processes, resulting in sudden changes in the IHTC. In the present research, a variable contact pressure trend was revealed by data mining an existing data base of verified FE simulation results to represent the exact contact pressure characteristics of hot stamping processes. Following a data driven approach, a dedicated, novel test scheme was developed and applied to determine the effect of contact pressure on the IHTC between a hot AA7075 aluminium alloy specimen and cold cast-iron tools in a single IHTC test, significantly improving testing efficiency.

\section{Development of a novel testing scheme for the characterisation of the contact pressure dependent IHTC}

The contact pressure distribution in hot stamping processes was generated by data mining an existing data base of verified hot stamping simulation results run under different forming conditions, collected by a cloud-based finite element (FE) simulation platform Smart Forming [6]. As shown in Fig. 1, the probability of contact pressure for all elements of the tools as a function of progression

\footnotetext{
* Corresponding author: liliang.wang@imperial.ac.uk
} 
of hot stamping process could be analysed to develop a novel testing scheme for the characterization of the contact pressure dependent IHTC to ensure that the exact contact pressure conditions encountered in hot stamping processes would be satisfied in the IHTC tests. The x-axis shown in Fig. 1 represented the normalized time periods of a hot stamping process rather than the actual time of a hot stamping process, leading to a dimensionless unit.

During the first time-period from 0 to 0.4 progression of hot stamping process, the contact area of the blank increased slowly from a small value and the contact pressure distribution was concentrated in the contact pressure range I from 0.4 to $0.8 \mathrm{MPa}$ in terms of probability. This indicated that the blank was being compressed between the blankholder and tools during the first time-period, resulting in a small blank area being contacted with the tools at small contact pressures. Therefore, the contact pressure range I determined the IHTC during the blankholding stage. During the second time-period from 0.4 to 0.75 progression of hot stamping process, the blank contact area increased rapidly. Meanwhile, the contact pressure range II from 2 to $7 \mathrm{MPa}$ started to increase and then had the largest probability when the progression of hot stamping process was larger than 0.5 . It was deduced that larger contact pressures were applied to deform the blank since the progression of hot stamping process of 0.4 . Therefore, the contact pressure range II determined the heat transfer between the blank and tools during the initial forming stage, i.e. the second time-period. During the third time-period from 0.7 to 1 progression of hot stamping process, the blank contact area increased dramatically to $100 \%$, indicating that the blank was continually deformed and subsequently quenched by the tools. The contact pressure range III from 12 to $25 \mathrm{MPa}$ had the largest probability during this time period, dominating the IHTC during the late forming and the subsequent initial quenching stages.

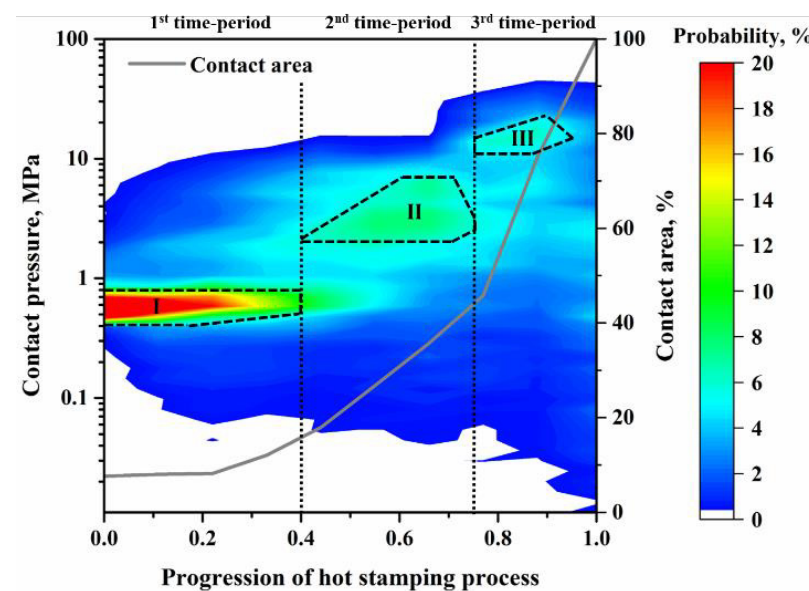

Fig. 1. The contact pressure distribution in the hot stamping processes.

Due to the contact pressure distribution being dominated by three contact pressure ranges, their average values of $0.6,4.5$ and $18.5 \mathrm{MPa}$ were the most representative contact pressures in the hot stamping processes. In order to represent the contact pressure characteristics of hot stamping processes, a variable contact pressure trend, suddenly increasing from 0.6 to $4.5 \mathrm{MPa}$ at 0.4 progression of hot stamping process and finally to 18.5 $\mathrm{MPa}$ at 0.75 progression of hot stamping process, could be used as a dedicated novel efficient testing scheme to determine the effect of contact pressure on the IHTC in a single IHTC test. Compared with previous studies, the novel test scheme developed by using the data driven approach for the characterisation of the contact pressure dependent IHTC would significantly improve testing efficiency.

\section{Determination of the IHTC using the dedicated novel testing scheme}

In the present research, an IHTC test facility was applied to simulate hot stamping processes [4]. A $120 \times 10 \times 2$ $\mathrm{mm}^{3}$ AA7075 specimen was screwed onto two blankholders, as shown in Fig. 2 (a), and heated by using direct resistance heating. Once the target temperature of $470^{\circ} \mathrm{C}$ was reached, the specimen was compressed by cold cast-iron punch and die at the pre-defined variable contact pressure, as shown in Fig. 3. A pair of thermocouple was embedded mid-thickness at the centre of the specimen to record its temperature histories during compression.

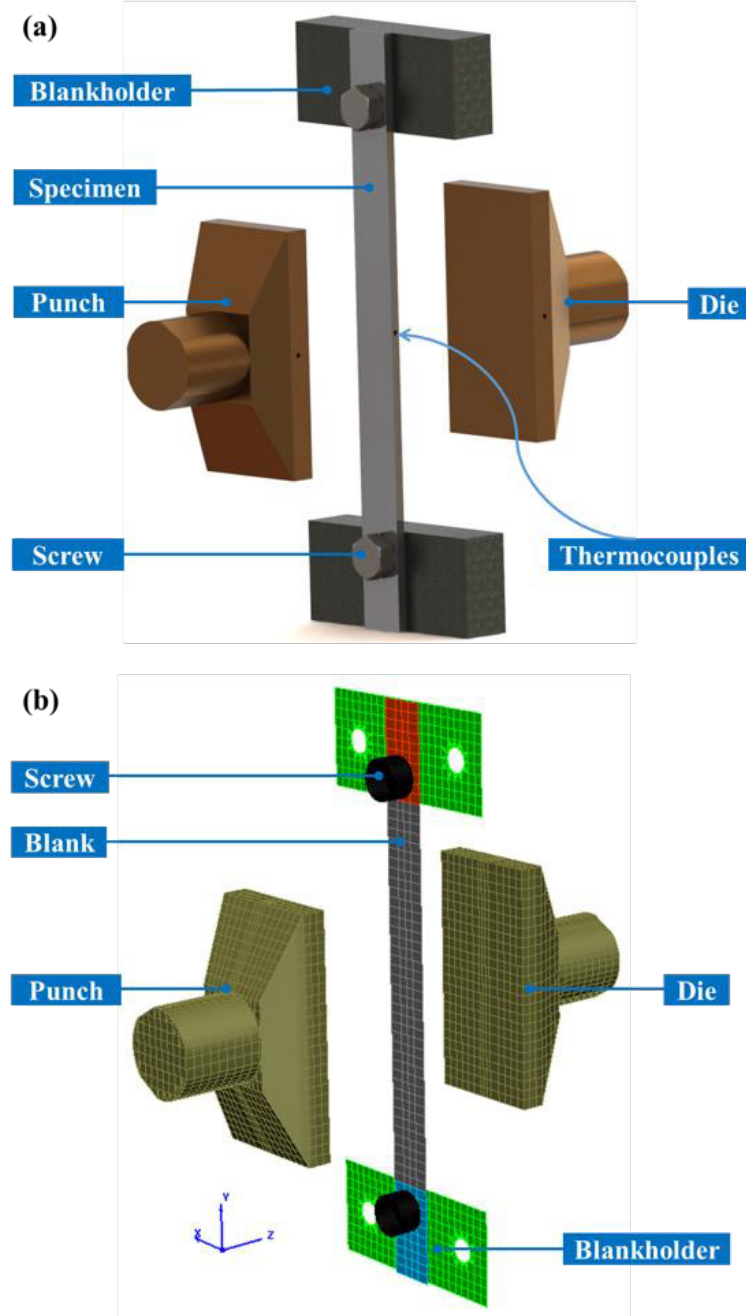

Fig. 2. (a) Overall schematic structure of the IHTC test facility; (b) The FE model of the IHTC test in PAM-STAMP. 
A FE model was built up in PAM-STAMP to simulate the IHTC tests, as shown in Fig. 2 (b), which was comprised of four stages. In the first gravity and holding stages, the specimen was located on the blankholders and fixed by the screws. In the subsequent stamping and quenching stages, the punch moved towards the specimen and compressed it against the die. A constant IHTC value was implemented between the specimen and tools to obtain the temperature evolution of the specimen during the first time-period, eliminating the effect of contact pressure on the IHTC. The simulated temperature evolution was then compared with the experimental results measured from the same location on the specimen to determine the IHTC value during the first time-period. Subsequently, different constant IHTC values were implemented in the second and third time-periods respectively to obtain the temperature evolution of the specimen after the first timeperiod. The best agreement between the experimental and simulated temperature evolutions identified the IHTC value during each time-period. Main simulation processing parameters were shown in Table 1.

Table 1. Main simulation processing parameters.

\begin{tabular}{|c|c|}
\hline Parameter & Value \\
\hline Initial specimen temperature & $470{ }^{\circ} \mathrm{C}$ \\
\hline Initial tool temperature & $25{ }^{\circ} \mathrm{C}$ \\
\hline Punch moving speed & $400 \mathrm{~mm} / \mathrm{s}$ \\
\hline Element size & $2 \mathrm{~mm}$ \\
\hline Element number of specimen & 240 \\
\hline Element number of tool & 325 \\
\hline Air convection coefficient & $20 \mathrm{~W} / \mathrm{m}^{2} \mathrm{~K}$ \\
\hline
\end{tabular}

\section{Results and discussions}

As shown in Fig. 3, the simulated temperature evolution of the specimen at a constant IHTC value of $2 \mathrm{~kW} / \mathrm{m}^{2} \mathrm{~K}$ agreed well with the experimental results during the first time-period from 0 to 0.4 seconds. When the contact pressure suddenly increased from 0.6 to $4.5 \mathrm{MPa}$ at 0.4 seconds, the temperature decreasing rate was significantly enhanced, resulting in a lager IHTC value of $7 \mathrm{~kW} / \mathrm{m}^{2} \mathrm{~K}$ during the second time-period. When the contact pressure increased to $18.5 \mathrm{MPa}$ at 0.75 seconds, the temperature decreasing rate was increased again. Due to the good agreement between the experimental and simulated temperature evolutions during the third time-period being achieved, the IHTC was considered to be increased to 11 $\mathrm{kW} / \mathrm{m}^{2} \mathrm{~K}$.
Therefore, the IHTC values at three different contact pressures were determined in a single IHTC test by using the dedicated novel test scheme, significantly improving testing efficiency. In addition, the determined IHTC values could represent the heat transfer between the blank and tools during the blankholding, forming and quenching stages.

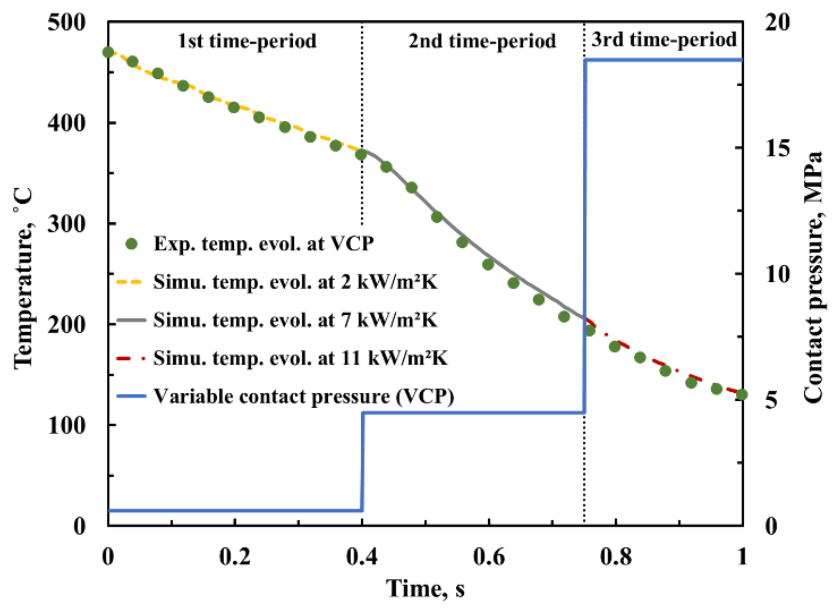

Fig. 3. The experimental and simulated temperature evolutions under variable contact pressure conditions.

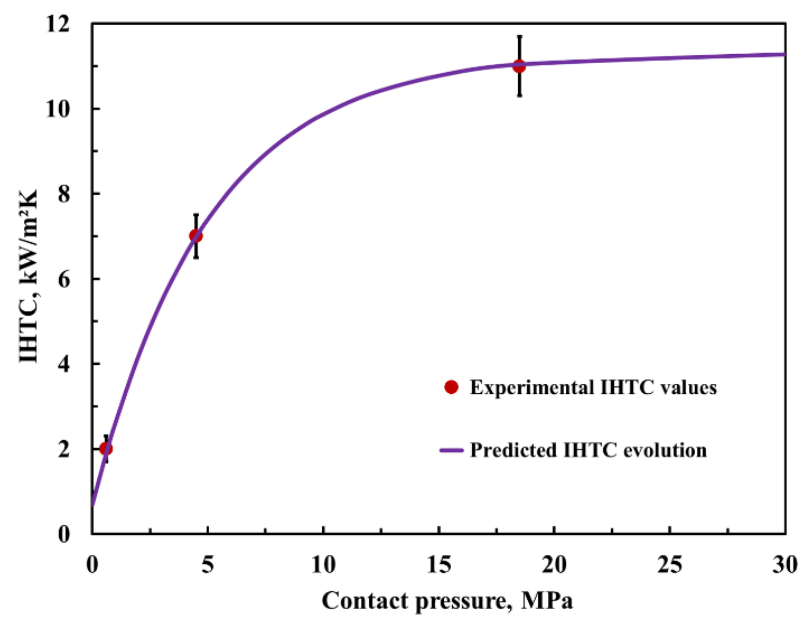

Fig. 4. The experimental and predicted IHTC evolutions with contact pressure.

As shown in Fig. 4, the IHTC increased dramatically from 2 to $7 \mathrm{~kW} / \mathrm{m}^{2} \mathrm{~K}$ when the contact pressure increased from 0.6 to $4.5 \mathrm{MPa}$, followed by a continuous increase to approximately $11 \mathrm{~kW} / \mathrm{m}^{2} \mathrm{~K}$ at $18.5 \mathrm{MPa}$. When the contact pressure was larger than $18.5 \mathrm{MPa}$, a plateau of the IHTC was observed. This was caused by the evolution of the real contact area between the specimen and tools with contact pressure. Due to the asperities on the contacting surfaces, the real contact area was less than the apparent contact area initially. The asperities on the hot specimen were deformed plastically and elastically by the cold tools during compression, resulting in the increasing real contact area [7] and thus higher IHTC values. When the real contact area approached the apparent contact area, the IHTC reached its peak value and thus the plateau was observed. 
The determined IHTC values were applied to the calibration of an experimentally verified IHTC model, which was developed in the study of Liu et al. [4], as shown in Eqs. (1-5).

$$
h=h_{a}+h_{c}
$$

The overall IHTC $h$ is a sum of two independent parts, the null-pressure IHTC $h_{a}$, representing the heat transfer across the air gap as a constant value of $0.7 \mathrm{~kW} / \mathrm{m}^{2} \mathrm{~K}$, and the solid-contact IHTC $h_{c}$, representing the heat transfer between the contacting solids when the contact pressure is applied.

$$
h_{c}=\alpha \cdot K_{s t} \cdot N_{P} / R_{s t}
$$

where $\alpha$ is a model parameter, $K_{s t}$ is the harmonic mean thermal conductivity of the contacting solids, $R_{s t}$ is the root mean square of surface roughness of the contacting solids, and $N_{P}$ is a pressure dependent parameter, which captures the effect of contact pressure on the IHTC.

$$
K_{s t}=2 \cdot k_{s} k_{t} /\left(k_{s}+k_{t}\right)
$$

where $k_{s}$ and $k_{t}$ are the thermal conductivities of the specimen and tools respectively.

$$
R_{s t}=\sqrt{R_{s}^{2}+R_{t}^{2}}
$$

where $R_{s}$ and $R_{t}$ are the surface roughnesses of the specimen and tools respectively.

$$
N_{P}=1-\exp \left(-\lambda \cdot P / \sigma_{U}\right)
$$

where $\lambda$ is a model parameter, $P$ is the applied contact pressure, and $\sigma_{U}$ is the ultimate strength of the specimen at the elevated temperature. Table 2 shows the material properties and calibrated parameters of the IHTC model.

Table 2. Material properties and model parameters of the IHTC model.

\begin{tabular}{|c|c|c|c|}
\hline$h_{a}$ & $k_{s}$ & $\sigma_{U}$ & $R_{s}$ \\
\hline $0.7 \mathrm{~kW} / \mathrm{m}^{2} \mathrm{~K}$ & $0.14 \mathrm{~kW} / \mathrm{mK}$ & $21 \mathrm{MPa}$ & $3.9 \mathrm{e}-7 \mathrm{~nm}$ \\
\hline$R_{t}$ & $k_{t}$ & $\alpha$ & $\lambda$ \\
\hline $1.8 \mathrm{e}-7 \mathrm{~nm}$ & $0.0352 \mathrm{~kW} / \mathrm{mK}$ & $8.1 \mathrm{e}-5$ & 4.2 \\
\hline
\end{tabular}

The IHTC evolution as a function of contact pressure was therefore predicted by the IHTC model, which could be implemented in the FE simulations to precisely simulate the temperature evolutions and cooling rates of components during hot stamping processes. This would be beneficial to the optimization of the hot stamping processing parameters and tool design to ensure that the critical cooling rates of the formed components were achieved. In addition, an excessive contact pressure could be prevented from being applied to reduce the tool wear and thus increase the tool life.

\section{Conclusions}

In the present research, a variable contact pressure trend was determined by data mining an existing data base of verified FE simulation results, collected by the cloudbased finite element (FE) simulation platform Smart Forming, in order to satisfy the contact pressure characteristics of hot stamping processes. Following the data driven approach, a dedicated, novel testing scheme was generated and applied to determine the IHTC values between a hot AA7075 aluminium alloy specimen and cold cast-iron tools, which were subsequently applied to the calibration of the experimentally verified IHTC model to predict the IHTC evolution as a function of contact pressure. Therefore, the effect of contact pressure on the IHTC was obtained in a single IHTC test by using the dedicated novel testing scheme, which has significantly improved testing efficiency.

The authors thank the European Commission for their support on the H2020 project 'Low Cost Materials Processing Technologies for Mass Production of Lightweight Vehicles (LoCoMaTech)', Grant No: H2020-NMBP-GV-2016(723517). This research was also supported by the China Scholarship Council (Grant CSC no. 201608060261): A non-profit institution enabling talented Chinese students to undertake a $\mathrm{PhD}$ programme overseas.

\section{References}

1. M. Raugei, O. El Fakir, L. Wang, J. Lin, D. Morrey, J. Clean. Prod. 83, 80 (2014)

2. Y. Chang, X. Tang, K. Zhao, P. Hu, Y. Wu, J. Mater. Process. Technol. 228, 25 (2016)

3. E. Caron, K. J. Daun, M. A. Wells, Int. J. Heat Mass Transf. 71, 396 (2014)

4. X. Liu, K. Ji, O. El Fakir, H. Fang, M. M. Gharbi, L. Wang, J. Mater. Process. Technol. 247, 158 (2017)

5. Z. Zhang, X. Li, Y. Zhao, X. Li Metall. Mater. Trans. B Process Metall. Mater. Process. Sci. 45, 1192 (2014)

6. Smartforming, http://smartforming.com/ (2016)

7. B. Buchner, M. Buchner, B. Buchmayr, Tribol. Int. 42, 897 (2009) 